\title{
IMPACT OF INNOVATIONS ON THE SAVING OF THE PRODUCTION COMPANY'S WORKING TIME FUND
}

\begin{abstract}
The article deals with innovation management in the conditions of a manufacturing company whose aim is to reduce the working time fund that directly determines productivity or efficiency of the company in competitive market conditions. Based on explicit quantification of time frames based on an analytical - chronometric method applicable to production operations in the process. The results of observation, time measurement, research and evaluation of time consumption during the implementation of a repeated production operation, or its complex part within defined production site are presented. Based on explicitly performed quantitative analysis, introducing of innovative technology, innovative solutions in the field of production technology management that support sustainable development with an emphasis on the development of environmental quality are presented including an explicit quantification of working time fund savings through the implementation of innovative machinery and equipment in critical production operations of the analysed production process.
\end{abstract}

Keywords: innovation, saving time, critical points of the production process.

\section{INTRODUCTION}

Innovation is a way of life of the company that intervenes in all partial components of the reproduction process (marketing, product and technology development, planning, production, sales, staff training, management, etc.) (Kováč et al., 2011). If the company identifies with the innovation strategy, then it is characterized by a high share of innovative products in the sales structure, the use of advanced technology with innovative management

\footnotetext{
${ }^{1}$ Alexander Tokarčík, MSc., PhD, Energy Cluster of Prešov Region, Slovak Republic; e-mail: klasterekpk@gmail.com

${ }^{2}$ Henrieta Pavolová, Assoc. prof., MSc., PhD, Technical University of Košice, Faculty of Mining, Ecology, Process Control and Geotechnologies, Letná 9, 04200 Košice, Slovak Republic; e-mail: henrieta.pavolova@tuke.sk (corresponding author). ORCID: 0000-0002-6507-0486.

${ }^{3}$ Tomáš Bakalár, Assoc. prof., MSc., PhD, Technical University of Košice, Faculty of Mining, Ecology, Process Control and Geotechnologies, Letná 9, 04200 Košice, Slovak Republic; e-mail: tomas.bakalar@tuke.sk. ORCID: 0000-0002-6985-9935.

${ }^{4}$ Lucia Bednárová, Assoc. prof. Ing. PhD, Technical University of Košice, Faculty of Mining, Ecology, Process Control and Geotechnologies, Letná 9, 04200 Košice, Slovak Republic; e-mail: lucia.bednarova@tuke.sk. ORCID: 0000-0002-8582-0643.
} 
(Dicová, 2008, Carter et al., 2001). For customers in the market (Estélyiová, 2007). The aim of innovation in the company is to increase sales to customers and to ensure quality as well as productivity towards the inside of the company, or efficiency. Subsequently, these facts are reflected in the philosophy of the company and its brand (Damanpour, Gopalakrishnan, 2001). In terms of costs, technology innovation is more demanding than product innovation, but in terms of market dominance, it is necessary to combine these innovations. Innovation in companies has its specifics presented in the form of risk, which is associated with the vision of success, the danger of failure and loss (Henriques, Sadorsky, 2007). Customer-oriented innovations need a source of ideas for new products and services, which are based primarily on their own analysis of the relevant market segment and customer behaviour in it (Ivanová, Tomanová, 2014). Therefore, companies in the region are concentrated in clusters, where they use their specific environment and available professional capacities.

By analysing the company's position in the market, it is possible to understand its overall situation in comparison with similar business entities (Vivero, 2002). The results then help to identify reserves related to innovations in the company and point to the positive development of the company's activities in reducing energy intensity. An important step in the process of increasing innovation is to focus on extensive data collection on production processes, as well as to analyse the individual production operations that take place on them (Budziakowski, 2016).

\section{AIM, SCOPE AND SUBJECT MATTER}

The aim of the case study was an exact analysis of the time consumption of partial production operations of the production process in the real conditions of the production company. Based on a detailed analysis of the time consumption of partial production operations, critical points of the production process of the final product were identified. In terms of identifying critical areas of the working time fund, production innovations of mechanical and technical equipment were proposed, the implementation of which would save the working time of the production process with an emphasis on maintaining the competitiveness of the production company, increasing productivity and environmental responsibility in accordance with the principles of sustainable development.

\section{RESEARCH METHODOLOGY}

For a detailed analysis of partial production operations in the production process and the normative of time consumption, the measurement was performed directly at the workplace using the method of analysis - chronometry. The time frame of the operation (chronometry) was used to determine the production time for individual production operations. The time frame of the operation is a part of working frames and captures the results of observation, measurement, examination and evaluation of time consumption when performing a certain repetitive production operation, or part of it at the workplace of an individual. Time consumption was measured using a stopwatch. During the analysis of a specific production process, its partial production operations were identified, which is illustrated in detail in Figure 1. 


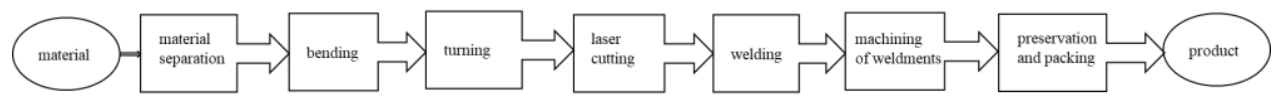

Figure 1. Production process in the analysed company

Source: Authors' own elaboration.

For the needs of compiling partial operations, the consumption of working hours of individual production operations was quantified by the analysis - chronometric method, based on which innovations of the production process were designed in the form of mechanical - technical equipment operating on the basis of systemic understanding of environmentally responsible company.

\section{RESULTS AND DISCUSSION}

For the analysed production process consisting of partial production operations (Figure 1), time frames with exactly identified time consumptions of individual work operations were constructed as follows:

- Time frame of the material separation, which uses a high-performance automatic band saw BOMAR transverse 610.440 GA with a cutting range up to 610 x $440 \mathrm{~mm}$ (Table 1).

Table 1. Material cutting by automatic saw

\begin{tabular}{|c|c|c|c|c|c|c|c|c|c|c|}
\hline \multirow{2}{*}{ Operation } & \multicolumn{10}{|c|}{ Measurements [min] } \\
\hline & M1 & M2 & M3 & M4 & M5 & M6 & M7 & M8 & M9 & M10 \\
\hline 1 & 6 & 7.23 & 7.49 & 7.33 & 10.38 & 9.14 & 9.39 & 8.44 & 8.91 & 8.63 \\
\hline 2 & 10.25 & 9.36 & 8.56 & 9.68 & 8.05 & 8.85 & 9.01 & 9.89 & 8.78 & 8.97 \\
\hline 3 & 15 & 14.69 & 15.67 & 15.77 & 15.76 & 18.45 & 17.54 & 18.23 & 15.89 & 15.92 \\
\hline 4 & 6.12 & 6.05 & 5.61 & 5.56 & 6.15 & 6.54 & 5.24 & 6.67 & 5.57 & 5.56 \\
\hline 5 & 1.74 & 1.83 & 1.83 & 1.74 & 1.95 & 2.01 & 1.35 & 1.29 & 2.13 & 2.58 \\
\hline$\Sigma$ & 39.11 & 39.16 & 39.2 & 40.08 & 42.3 & 45 & 42.5 & 44.5 & 41.3 & 41.7 \\
\hline Total measurement time & 414.78 & \multirow{2}{*}{$=$} & \multirow{2}{*}{\multicolumn{8}{|c|}{ Average measurement time 41.478 minute per piece }} \\
\hline Number of measurements & 10 & & & & & & & & & \\
\hline $\begin{array}{l}\text { Maximum measurement } \\
\text { time: }\end{array}$ & \multicolumn{2}{|c|}{$44.99 \mathrm{~min}}$. & \multicolumn{8}{|c|}{ Minimum measurement time: $39.11 \mathrm{~min}$. } \\
\hline $\mathrm{U}_{\mathrm{A}}$ standard uncertainty: & 0.26 & Precision: & $8.47 \%$ & & & & $-5.71 \%$ & & & \\
\hline \multicolumn{11}{|c|}{ Time consumption $=0.69$ hour per piece } \\
\hline
\end{tabular}

Notes: 1 - Loading drawing documentation, 2 - Material selection, 3 - Sawing, 4 - Control of separated material, 5 - Material storage.

Source: Authors own elaboration.

- Ermak Speed bend material bending time frame, which provides bending with the required accuracy and speed, while repeatable accuracy is possible thanks to the synchronization of the hydraulic cylinders with the servo-valves, and automatic axis indexing at machine start-up calibration (Table 2). 
Table 2. Material bending with Ermak Speed bend

\begin{tabular}{|c|c|c|c|c|c|c|c|c|c|c|}
\hline \multirow{2}{*}{ Operation } & \multicolumn{10}{|c|}{ Measurements [min] } \\
\hline & M1 & M2 & M3 & M4 & M5 & M6 & M7 & M8 & M9 & M10 \\
\hline 1 & 3.34 & 3.47 & 3.56 & 3.39 & 3.45 & 3.3 & 3.37 & 3.44 & 3.43 & 3.39 \\
\hline 2 & 10.61 & 10.34 & 10.65 & 10.3 & 10.41 & 9.85 & 10.18 & 10.22 & 10.18 & 10.24 \\
\hline 3 & 10.66 & 10.35 & 10.53 & 10.2 & 10.39 & 9.76 & 10.12 & 10.28 & 10.19 & 10.22 \\
\hline 4 & 1.01 & 1.86 & 1.87 & 1.76 & 1.85 & 1.72 & 1.83 & 1.83 & 1.75 & 1.84 \\
\hline 5 & 2.23 & 1.72 & 1.64 & 2.02 & 1.98 & 1.97 & 2.31 & 1.96 & 2.22 & 1.97 \\
\hline 6 & 1.45 & 1.1 & 1.13 & 1.17 & 1.15 & 1.08 & 1.18 & 1.05 & 1.22 & 1.12 \\
\hline 7 & 0.62 & 0.71 & 0.58 & 0.62 & 0.68 & \begin{tabular}{|l|}
0.76 \\
\end{tabular} & 0.66 & 0.77 & 0.69 & 0.65 \\
\hline 8 & 15.04 & 15.12 & 15.15 & 14.84 & 14.96 & 14.43 & 14.63 & 14.92 & 14.62 & 14.4 \\
\hline 9 & 0.48 & 0.64 & 0.48 & 0.82 & 0.43 & 1.18 & 0.6 & 0.63 & 0.59 & 0.6 \\
\hline 10 & 0.21 & 0.21 & 0.24 & 0.36 & 0.26 & \begin{tabular}{|l|}
0.26 \\
\end{tabular} & 0.33 & 0.34 & 0.27 & 0.22 \\
\hline 4 & 1.88 & 1.86 & 1.87 & 1.76 & 1.85 & 1.72 & 1.83 & 1.86 & 1.75 & 1.84 \\
\hline 6 & 1.14 & 1.1 & 1.13 & 1.17 & 1.15 & 1.04 & 1.18 & 1.05 & 1.12 & 1.09 \\
\hline 7 & 0.65 & 0.69 & 0.48 & 0.61 & 0.64 & 0.74 & 0.72 & 0.68 & 0.71 & 0.69 \\
\hline 8 & 14.84 & 14.82 & 15.05 & 14.64 & 15.12 & 14.48 & 14.83 & 14.92 & 15.12 & 14.51 \\
\hline 9 & 0.52 & 0.48 & 0.62 & 0.82 & 0.75 & 1.09 & 0.94 & 0.83 & 1.08 & 0.97 \\
\hline 10 & 0.19 & 0.24 & 0.23 & 0.37 & 0.39 & 0.34 & 0.38 & 0.31 & 0.34 & 0.29 \\
\hline 11 & 14.88 & 16.39 & 18.02 & 14.62 & 14.13 & 15.16 & 18.19 & 17.83 & 19.57 & 14.36 \\
\hline 12 & 0.12 & 0.11 & 0.12 & 0.13 & 0.11 & 0.15 & 0.13 & 0.12 & 0.13 & 0.14 \\
\hline$\Sigma$ & 79.87 & 81.21 & \begin{tabular}{|l|}
83.35 \\
\end{tabular} & 79.6 & 79.7 & 79.03 & 83.41 & 83.04 & 84.98 & 78.54 \\
\hline $\begin{array}{l}\text { Total measurement } \\
\text { time }\end{array}$ & 812.73 & \multirow{2}{*}{$=$} & \multirow{2}{*}{\multicolumn{8}{|c|}{ Average measurement time 81,273 minute per piece }} \\
\hline $\begin{array}{l}\text { Number of } \\
\text { measurements }\end{array}$ & 10 & & & & & & & & & \\
\hline $\begin{array}{l}\text { Maximum } \\
\text { measurement time: }\end{array}$ & $84.98 \mathrm{~min}$. & & \multicolumn{8}{|c|}{ Minimum measurement time: 78,54 min. } \\
\hline $\begin{array}{l}\text { UA standard } \\
\text { uncertainty: }\end{array}$ & 0.08 & Precision: & $4.56 \%$ & \multicolumn{7}{|c|}{$-3.36 \%$} \\
\hline
\end{tabular}

Notes: 1 - Loading drawing documentation, 2 - Creating a bending program, 3 - Tool selection against material, 4 - Material inspection - dimensionality, 5-Attaching the tool to the press brake, 6 - Starting the program, 7 - Inserting material, 8 - Ram lower $=>$ zoom bend, 9 - Gauge bending check, 10 - Gauge bending check, 11 - Bending repair - product finalization, 12 - Product storage.

Source: Authors own elaboration.

- Turning time frame using a TOS SV 18 RA type lathe, which is a manual, universal centre lathe and represents a highly precise and powerful machine, well usable especially in small series and piece production (Table 3 ).

- Laser cutting time frame, which uses two types of cutting gases, oxygen and nitrogen. During oxygen laser cutting, the material is burned and an oxidized layer unsuitable for surface treatment is formed on its edges. During nitrogen cutting, the material melts, which is then blown out of the dividing track (Table 4).

- Welding time frame, which is the process of making a permanent joint by forming interatomic bonds between the welded parts during heating or plastic deformation or by the action of both (Table 5). 
Table 3. Turning

\begin{tabular}{|c|c|c|c|c|c|c|c|c|c|c|}
\hline \multirow{2}{*}{ Operation } & \multicolumn{10}{|c|}{ Measurements [min] } \\
\hline & M1 & M2 & M3 & M4 & M5 & M6 & M7 & M8 & M9 & M10 \\
\hline 1 & 2.85 & 2.34 & 2.49 & 2.68 & 2.94 & 2.62 & 2.7 & 2.44 & 2.58 & 3.02 \\
\hline 2 & 8.52 & 10.01 & 8.09 & 9.42 & 8.98 & 9.24 & 8.85 & 8.78 & 8.75 & 8.97 \\
\hline 3 & 2.27 & 2.42 & 2.43 & 2.43 & 2.48 & 2.46 & 2.42 & 2.38 & 2.46 & 2.58 \\
\hline 4 & 4.64 & 4.59 & 4.58 & 4.58 & 4.48 & 4.38 & 4.61 & 4.82 & 4.59 & 4.48 \\
\hline 5 & 17.44 & 16.95 & 15.71 & 14.93 & 15.56 & 16.84 & 16.82 & 17.19 & 17.64 & 16.59 \\
\hline 6 & 1.44 & 1.94 & 3.09 & 2.57 & 1.84 & 2.05 & 2.93 & 3.12 & 2.72 & 2.35 \\
\hline 7 & 3.35 & 2.98 & 3.51 & 3.43 & 3.45 & 3.27 & 3.15 & 3.42 & 3.15 & 3.28 \\
\hline 8 & 6.57 & 6.7 & 6.84 & 6.69 & 6.74 & 6.54 & \begin{tabular}{|l|}
6.51 \\
\end{tabular} & 6.82 & 6.72 & 6.75 \\
\hline 9 & 1.94 & 2.33 & 1.76 & 2.47 & 2.34 & 2.18 & 1.98 & 1.78 & 1.99 & 1.97 \\
\hline 10 & 3.35 & 3.45 & 3.35 & 3.42 & 3.23 & 3.54 & 3.48 & 3.18 & 3.24 & 3.35 \\
\hline 9 & 1.94 & 2.33 & 1.76 & 2.47 & 2.3 & 2.1 & 1.87 & 1.71 & 1.9 & 1.95 \\
\hline 11 & 3.35 & 3.17 & 3.35 & 3.34 & 3.23 & 3.13 & 3.13 & 3.17 & 3.13 & 3.08 \\
\hline 12 & 0.49 & 0.36 & 0.42 & 0.3 & 0.37 & 0.34 & 0.37 & 0.34 & 0.32 & 0.42 \\
\hline 13 & 0.44 & 0.47 & 0.47 & 0.51 & 0.46 & 0.46 & 0.47 & 0.45 & 0.47 & 0.46 \\
\hline 14 & 1.37 & 1.48 & 1.39 & 1.41 & 1.45 & 1.39 & 1.34 & 1.41 & 1.39 & 1.45 \\
\hline 15 & 0.43 & 0.48 & 0.45 & 0.49 & 0.43 & 0.45 & 0.48 & 0.42 & 0.44 & 0.42 \\
\hline$\Sigma$ & 60.39 & 62 & 59.69 & 61.08 & 60.29 & 60.99 & 61.11 & 61.42 & 61.42 & 61.13 \\
\hline Total measureme & 609.57 & \multirow[b]{2}{*}{$=$} & \multirow{2}{*}{\multicolumn{8}{|c|}{ Average measurement time 60.957 minute per piece }} \\
\hline $\begin{array}{l}\text { Number of } \\
\text { measurements }\end{array}$ & 10 & & & & & & & & & \\
\hline $\begin{array}{l}\text { Maximum measurement } \\
\text { time: }\end{array}$ & $62.00 \mathrm{~min}$. & & \multicolumn{8}{|c|}{ Minimum measurement time: 59.69 min. } \\
\hline UA standard uncertainty: & 0.07 & Precision: & $1.71 \%$ & & & & $-2.09 \%$ & & & \\
\hline
\end{tabular}

Notes: 1 - Loading drawing documentation, 2 - Semi-finished product inspection, 3 - Material clamping, 4 - Cutting tool selection, 5 - Tool clamping 6 - Zoom tool, 7 - Turning, 8 - Product rotation, 9 -Zoom tool, 10-Turning, 11 - Drilling, 12 - Termination of activities on the facility, 13 - Product removal, 14 - Product measurement and control, 15 - Product storage.

Source: Authors own elaboration.

Table 4. Laser cutting

\begin{tabular}{|c|c|c|c|c|c|c|c|c|c|c|}
\hline \multirow{2}{*}{ Operation } & \multicolumn{10}{|c|}{ Measurements [min] } \\
\cline { 2 - 13 } & M1 & M2 & M3 & M4 & M5 & M6 & M7 & M8 & M9 & M10 \\
\hline $\mathbf{1}$ & 12.5 & 12.87 & 14.22 & 14.94 & 16.61 & 14.63 & 15.03 & 13.51 & 14.26 & 13.81 \\
\hline $\mathbf{2}$ & 46.81 & 55.06 & 46.01 & 52.51 & 50.74 & 51.59 & 49.25 & 48.61 & 48.62 & 49.93 \\
\hline $\mathbf{3}$ & 13.41 & 12.31 & 13.87 & 12.32 & 14.01 & 13.74 & 12.48 & 13.18 & 13.6 & 14.36 \\
\hline $\mathbf{4}$ & 26.65 & 25.68 & 26.58 & 25.49 & 25.84 & 24.86 & 25.98 & 26.67 & 25.61 & 24.89 \\
\hline $\mathbf{5}$ & 10.91 & 9.83 & 9.86 & 9.28 & 10.49 & 10.8 & 11.92 & 10.3 & 10.74 & 10.97 \\
\hline $\mathbf{6}$ & 16.88 & 15.87 & 17.64 & 14.33 & 13.22 & 15.45 & 16.31 & 17.28 & 15.04 & 14.08 \\
\hline $\mathbf{7}$ & 61.54 & 61.34 & 60.04 & 60.48 & 60.49 & 61.26 & 60.53 & 62.04 & 61.59 & 60.89 \\
\hline $\mathbf{5}$ & 18.14 & 17.42 & 19.12 & 18.62 & 18.25 & 17.46 & 17.41 & 17.52 & 17.29 & 17.13 \\
\hline $\mathbf{6}$ & 1.18 & 1.82 & 1.05 & 1.77 & 1.22 & 1.17 & 1.02 & 1.86 & 1.25 & 1.59 \\
\hline $\mathbf{7}$ & 76.48 & 77.58 & 76.84 & 76.98 & 78.02 & 78.06 & 77.68 & 78.04 & 77.95 & 78.88 \\
\hline
\end{tabular}


Table 4 (cont.). Laser cutting

\begin{tabular}{|c|c|c|c|c|c|c|c|c|c|c|}
\hline \multirow{2}{*}{ Operation } & \multicolumn{10}{|c|}{ Measurements [min] } \\
\hline & M1 & M2 & M3 & M4 & M5 & M6 & M7 & M8 & M9 & M10 \\
\hline 5 & 13.34 & 12.81 & 12.02 & 13.74 & 12.99 & 11.71 & 10.41 & 11.44 & 11.49 & 12.84 \\
\hline 6 & 1.14 & 1.99 & 2.39 & 1.65 & 2.1 & \begin{tabular}{|l|}
1.91 \\
\end{tabular} & \begin{tabular}{|l|}
2.05 \\
\end{tabular} & 1.88 & 1.78 & 2.36 \\
\hline 7 & 26.76 & 28.48 & 29.13 & 25.16 & 24.98 & 28.77 & 29.44 & 27.33 & 29.02 & 27.58 \\
\hline 8 & 7.57 & 6.58 & 6.92 & 7.24 & 7.98 & 6.84 & 7.05 & 6.92 & 6.97 & 7.34 \\
\hline$\Sigma$ & 333.31 & 339.63 & 335.7 & 334.5 & 336.94 & 338.3 & 336.6 & 336.6 & 335.2 & 336.7 \\
\hline Total measurement time & 3363.32 & \multirow[b]{2}{*}{$=$} & \multirow{2}{*}{\multicolumn{8}{|c|}{ Average measurement time 336.332 minute per piece }} \\
\hline $\begin{array}{l}\text { Number of } \\
\text { measurements }\end{array}$ & 10 & & & & & & & & & \\
\hline $\begin{array}{l}\text { Maximum measurement } \\
\text { time: }\end{array}$ & \multicolumn{2}{|c|}{$339.32 \mathrm{~min}}$. & \multicolumn{8}{|c|}{ Minimum measurement time: $333.31 \mathrm{~min}$. } \\
\hline $\mathrm{U}_{\mathrm{A}}$ standard uncertainty: & 0.26 & Precision: & $0.98 \%$ & \multicolumn{7}{|c|}{$-0.90 \%$} \\
\hline Time consumption $=5.6$ & our per & piece & & & & & & & & \\
\hline
\end{tabular}

Notes: 1 - Creation of technological process, 2 - Programming, 3 - Material typing, 4 - Creation of technological process, 5 - Material selection, 6 - Material focus, 7 - Laser cutting, 8 - Product storage and surplus.

Source: Authors own elaboration.

Table 5. Welding

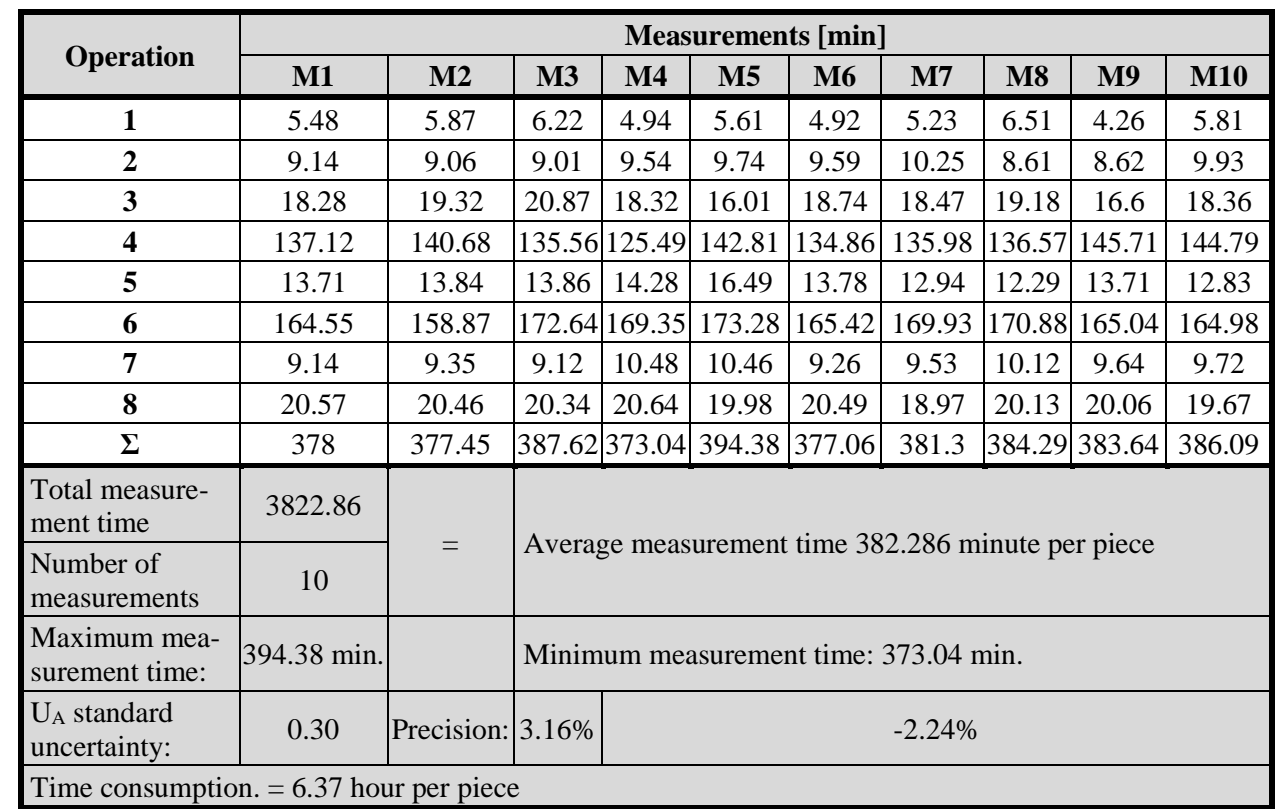

Notes: 1 - Loading drawing documentation, 2 - Inspection and preparation of components, 3 - Clamping parts, 4 - Scoring, 5 - Control, 6 - Welding, 7 - Dimension check, 8 - Interoperational inspection and Material storage.

Source: Authors own elaboration. 
- A precision machining time frame that is a collection of technological processes that change the appearance and functionality of a metal part to modify its properties, extend functionality, or modify design. This production operation was carried out using WHN 13 CNC, which is a universal CNC machine for precision horizontal milling, coordinate drilling and boring, thread cutting for box, plate and complex work pieces weighing up to $12,000 \mathrm{~kg}$ (Table 6).

- Time frame of preservation and packaging, which achieves the protection of all metal parts during transport and storage at the customer (Table 7).

Table 6. Precise machining

\begin{tabular}{|c|c|c|c|c|c|c|c|c|c|c|}
\hline \multirow{2}{*}{ Operation } & \multicolumn{10}{|c|}{ Measurements [min] } \\
\hline & M1 & M2 & M3 & M4 & M5 & M6 & M7 & M8 & M9 & M10 \\
\hline 1 & 30.12 & 32.81 & 30.98 & 29.76 & 30.45 & 31.83 & 30.64 & 30.99 & 31.97 & 30.76 \\
\hline 2 & 50.23 & 52.48 & 51.54 & 51.83 & 49.82 & 50.94 & 51.22 & 48.98 & 50.34 & 50.98 \\
\hline 3 & 10.73 & 10.02 & 9.24 & 11.64 & 16.54 & 14.4 & 10.81 & 11.88 & 12.67 & 10.84 \\
\hline 4 & 60.37 & 63.72 & 59.28 & 58.76 & 61.82 & 63.83 & 60.74 & 64.72 & 62.21 & 59.88 \\
\hline 5 & 10.71 & 10.29 & 12.41 & 11.92 & 12.63 & 9.81 & 9.43 & 9.68 & 10.91 & 11.49 \\
\hline 6 & 6.16 & 6.62 & 7.75 & 8.83 & 8.86 & 7.64 & 7.94 & 8.34 & 8.46 & 9.12 \\
\hline 7 & 16.76 & 16.28 & 17.95 & 17.63 & 18.14 & 18.46 & 16.12 & 17.65 & 17.48 & 18.46 \\
\hline 8 & 8.13 & 9.63 & 8.06 & 8.9 & 9.34 & 8.92 & 9.63 & 9.77 & \begin{tabular}{|l|}
8.54 \\
\end{tabular} & 9.34 \\
\hline 9 & 5.98 & 5.74 & 7.87 & 9.53 & 8.56 & 8.82 & 8.36 & 7.84 & 8.42 & 9.41 \\
\hline 10 & 4.9 & 5.32 & 4.83 & 6.41 & 6.41 & 7.63 & 8.12 & 5.18 & 6.03 & 7.12 \\
\hline 6 & 5.16 & 5.62 & 7.35 & 5.36 & 6.23 & 5.26 & 7.9 & 6.34 & 5.66 & 6.81 \\
\hline 7 & 55.76 & 56.28 & 59.68 & 57.41 & 52.28 & \begin{tabular}{|l|}
54.76 \\
\end{tabular} & 56.81 & 57.65 & \begin{tabular}{|l|}
57.48 \\
\end{tabular} & 54.76 \\
\hline 8 & 15.13 & 16.64 & 16.94 & 15.89 & 15.01 & 15.24 & 16.46 & 15.88 & 16.16 & 15.41 \\
\hline 9 & 10.94 & 10.74 & 9.87 & 10.93 & 9.98 & \begin{tabular}{|l|}
12.23 \\
\end{tabular} & 9.92 & 12.34 & \begin{tabular}{|l|}
10.73 \\
\end{tabular} & 10.16 \\
\hline 10 & 6.95 & 7.32 & 8.81 & 5.98 & 6.15 & 7.58 & 5.67 & 7.34 & 6.83 & 6.12 \\
\hline 6 & 5.23 & 5.62 & 7.35 & 5.63 & 8.45 & 7.84 & 5.01 & 3.95 & 6.63 & 6.14 \\
\hline 7 & 30.63 & 31.28 & 30.86 & 42.02 & 39.2 & 43.94 & 30.42 & 33.81 & \begin{tabular}{|l|}
32.88 \\
\end{tabular} & 37.67 \\
\hline 8 & 12.73 & 14.64 & \begin{tabular}{|l|}
13.94 \\
\end{tabular} & 12.21 & 14.12 & \begin{tabular}{|l|}
11.34 \\
\end{tabular} & 12.83 & 11.74 & \begin{tabular}{|l|}
16.97 \\
\end{tabular} & 13.21 \\
\hline 9 & 7.89 & 8.74 & 7.83 & 8.23 & 7.78 & 8.54 & 7.81 & 7.48 & 8.64 & 8.21 \\
\hline 10 & 11.97 & 12.32 & 14.83 & 13.41 & 18.95 & 12.63 & 13.64 & 11.95 & \begin{tabular}{|l|}
12.36 \\
\end{tabular} & 11.46 \\
\hline 6 & 5.67 & 6.62 & 7.35 & 11.36 & 9.24 & 6.26 & 8.74 & 6.81 & \begin{tabular}{|l|}
7.75 \\
\end{tabular} & 7.48 \\
\hline 7 & 31.03 & 66.28 & 29.88 & 32.31 & 34.28 & 36.34 & 31.92 & 32.65 & \begin{tabular}{|l|}
33.78 \\
\end{tabular} & 32.54 \\
\hline 8 & 12.34 & 12.63 & 11.94 & 11.85 & 12.4 & 11.34 & 11.83 & 12.36 & 11.84 & 13.42 \\
\hline 9 & 7.94 & 8.74 & 7.73 & 9.53 & 8.76 & 9.12 & 7.63 & 8.14 & 8.18 & 8.035 \\
\hline 10 & 10.95 & 9.36 & 11.83 & 8.4 & 9.92 & \begin{tabular}{|l|}
12.41 \\
\end{tabular} & 11.94 & 12.14 & \begin{tabular}{|l|}
11.98 \\
\end{tabular} & 9.87 \\
\hline 6 & 5.64 & 5.62 & 7.35 & 6.36 & 5.34 & 5.56 & 5.83 & 7.36 & 7.78 & 6.81 \\
\hline 7 & 31.02 & 36.21 & 39.86 & 32.41 & 30.12 & 29.92 & 32.53 & 31.14 & \begin{tabular}{|l|}
29.58 \\
\end{tabular} & 29.74 \\
\hline 8 & 11.73 & 13.64 & 16.45 & 14.72 & 33.72 & 12.67 & 11.93 & 12.34 & 11.94 & 12.63 \\
\hline 9 & 7.98 & 8.48 & \begin{tabular}{|l|}
7.87 \\
\end{tabular} & 8.16 & 7.99 & 7.57 & 7.59 & 8.71 & \begin{tabular}{|l|}
8.63 \\
\end{tabular} & 7.94 \\
\hline 10 & 12.59 & 11.36 & \begin{tabular}{|l|}
12.83 \\
\end{tabular} & 13.16 & 14.62 & \begin{tabular}{|l|}
13.35 \\
\end{tabular} & 11.34 & 18.58 & \begin{tabular}{|l|}
18.35 \\
\end{tabular} & 17.61 \\
\hline 11 & 9.77 & 9.78 & 9.35 & 8.76 & 11.28 & 10.86 & 17.34 & 11.94 & \begin{tabular}{|l|}
11.85 \\
\end{tabular} & 12.47 \\
\hline 12 & 2.51 & 2.56 & 2.62 & 2.17 & 3.63 & 2.94 & 3.85 & 3.56 & 3.73 & 3.64 \\
\hline
\end{tabular}


Table 6 (cont.). Precise machining

\begin{tabular}{|c|c|c|c|c|c|c|c|c|c|c|}
\hline \multirow{2}{*}{ Operation } & \multicolumn{10}{|c|}{ Measurements [min] } \\
\hline & M1 & M2 & M3 & M4 & M5 & M6 & M7 & M8 & M9 & M10 \\
\hline 13 & 7.72 & 8.12 & 7.82 & 7.98 & 8.74 & 7.87 & 9.58 & 8.09 & 7.86 & 8.24 \\
\hline 14 & 12.56 & 13.31 & 12.45 & 13.95 & 14.32 & 12.83 & 13.4 & 11.26 & \begin{tabular}{|l|}
12.02 \\
\end{tabular} & 12.97 \\
\hline 15 & 3.46 & 2.61 & 2.54 & 2.7 & 3.97 & 3.35 & 3.63 & 2.43 & 2.53 & 2.43 \\
\hline$\Sigma$ & 539.39 & 597.43 & 575.24 & 576.1 & 608.56 & 584.03 & 568.56 & 571.02 & 571.02 & 573.175 \\
\hline $\begin{array}{l}\text { Total measure- } \\
\text { ment time }\end{array}$ & 5772.675 & \multirow[b]{2}{*}{$=$} & \multirow{2}{*}{\multicolumn{8}{|c|}{ Average measurement time 577.2675 minute per piece }} \\
\hline $\begin{array}{l}\text { Number of } \\
\text { measurements }\end{array}$ & 10 & & & & & & & & & \\
\hline $\begin{array}{l}\text { Maximum mea- } \\
\text { surement time: }\end{array}$ & $84.98 \mathrm{~min}$. & & \multicolumn{8}{|c|}{ Minimum measurement time: $539.39 \mathrm{~min}}$. \\
\hline $\begin{array}{l}\text { UA standard } \\
\text { uncertainty: }\end{array}$ & 0.57 & $\begin{array}{l}\text { Preci- } \\
\text { sion: }\end{array}$ & $5.42 \%$ & & & & $-6.56 \%$ & & & \\
\hline
\end{tabular}

Notes: 1 - Loading drawing documentation, 2 - Creating a drilling program, 3 - Tool selection against material, 4 - Weldment clamping, 5 - Measuring probe measurement, 6 - Inserting a machining tool, 7 - Execution of the program, 8 - Product release, 9 - Product position change, 10 - Measuring probe measurement, 11 - Control measurement, 12 - Product cleaning, 13 - Product release, 14 - Product transfer, 15 - Product storage.

Source: Authors own elaboration.

Table 7. Preservation and packaging

\begin{tabular}{|c|c|c|c|c|c|c|c|c|c|c|}
\hline \multirow{2}{*}{ Operation } & \multicolumn{10}{|c|}{ Measurements [min] } \\
\hline & M1 & M2 & M3 & M4 & M5 & M6 & M7 & M8 & M9 & M10 \\
\hline 1 & 15.02 & 14.86 & 14.97 & 15.45 & 14.76 & 15.48 & 14.77 & 15.12 & 15.03 & 15.24 \\
\hline 2 & 6.15 & 6.24 & 6.16 & 6.58 & 7.12 & 6.82 & 6.79 & 6.44 & 7.15 & 7.42 \\
\hline 5 & 10.12 & 10.45 & 9.94 & 10.23 & 10.41 & 10.15 & 9.93 & 9.75 & 10.2 & 9.84 \\
\hline$\Sigma$ & 31.29 & 31.55 & 31.07 & 32.26 & 32.29 & 32.45 & 31.49 & 31.31 & 32.38 & 32.5 \\
\hline Total measurement time & 318.59 & \multirow[b]{2}{*}{$=$} & \multirow{2}{*}{\multicolumn{4}{|c|}{ Average measurement time }} & \multirow{2}{*}{\multicolumn{4}{|c|}{31.859 minute per piece }} \\
\hline $\begin{array}{l}\text { Number of } \\
\text { measurements }\end{array}$ & 10 & & & & & & & & & \\
\hline $\begin{array}{l}\text { Maximum measurement } \\
\text { time: }\end{array}$ & $32.50 \mathrm{~min}$. & & \multicolumn{8}{|c|}{ Minimum measurement time: $31.07 \mathrm{~min}}$. \\
\hline $\mathrm{U}_{\mathrm{A}}$ standard uncertainty: & 0.02 & Precision: & $2.01 \%$ & \multicolumn{7}{|c|}{$-2.48 \%$} \\
\hline Time consumption $=0.5$ & our per pic & & & & & & & & & \\
\hline
\end{tabular}

Notes: 1 - Product cleaning, 2 - Preservative application, 3 - Packing.

Source: Authors own elaboration. 
Based on a detailed analysis of time frames of partial operations in terms of the analysis - chronometry method. identified critical points were identified and are included in Table 8:

- bending: a critical point in bending repair, i.e. product finalization,

- turning: critical points in partial production operations including turning the product and approaching the tool.

- precise machining: critical points in partial production operations including uncoupling the product. changing the position of the product and measuring with a measuring probe.

Table 8. Identification of critical points in the production process

\begin{tabular}{|c|c|c|c|c|c|c|c|c|c|c|c|}
\hline Manufacturing & \multicolumn{11}{|c|}{ Bending } \\
\hline $\begin{array}{c}\text { Recalculation } \\
\text { of critical points }\end{array}$ & M1 & M2 & M3 & M4 & M5 & M6 & M7 & M8 & M9 & M10 & $\begin{array}{c}\text { In } \\
\text { sum }\end{array}$ \\
\hline $11[\%]$ & 18.63 & 20.18 & 21.62 & 18.37 & 17.73 & 19.18 & 21.81 & 21.47 & 23.03 & 18.28 & $\begin{array}{c}20.07 \\
\%\end{array}$ \\
\hline $\begin{array}{c}\text { Manufacturing } \\
\text { operation }\end{array}$ & \multicolumn{11}{|c|}{ Turning } \\
\hline $\begin{array}{c}\text { Recalculation } \\
\text { of critical points }\end{array}$ & M2 & M3 & M4 & M5 & M6 & M7 & M8 & M9 & M10 & & $\begin{array}{c}\text { In } \\
\text { sum }\end{array}$ \\
\hline $8[\%]$ & 10.81 & 11.46 & 10.9 & 11.18 & 10.72 & 10.65 & 11.10 & 10.93 & 11.04 & & \multirow{2}{*}{$\begin{array}{c}14.37 \\
\%\end{array}$} \\
\hline $9[\%]$ & 3.7 & 2.95 & 4.04 & 3.88 & 3.57 & 3.24 & 2.90 & 3.24 & 3.22 & & \\
\hline $\begin{array}{c}\text { Manufacturing } \\
\text { operation }\end{array}$ & \multicolumn{11}{|c|}{ Precise machining } \\
\hline $\begin{array}{c}\text { Recalculation } \\
\text { of critical points }\end{array}$ & M1 & M2 & M3 & M4 & M5 & M6 & M7 & M8 & M9 & M10 & $\begin{array}{c}\text { In } \\
\text { sum }\end{array}$ \\
\hline $8[\%]$ & 11.13 & 11.24 & 11.7 & 11.03 & 13.9 & 10.19 & 11.02 & 10.87 & 11.3 & 11.17 & \multirow{3}{*}{$\begin{array}{c}27.84 \\
\%\end{array}$} \\
\hline $9[\%]$ & 7.55 & 7.1 & 7.16 & 8.05 & 7.08 & 7.92 & 7.27 & 7.79 & 7.7 & 7.63 & \\
\hline $10[\%]$ & 8.78 & 7.65 & 9.24 & 8.22 & 9.13 & $\begin{array}{l}9.18 \\
\end{array}$ & 8.92 & 9.67 & 9.59 & 9.1 & \\
\hline
\end{tabular}

Source: Authors own elaboration.

In view of the above, based on the identified critical points of the working time fund according to a clear quantification of partial production operations in terms of analytical chronometric method of measuring time frames it can be concluded that individual machinery should be implemented in the above production operations:

- LVD EasyForm9 220/30 press brake in the identified critical bending operation,

- CNC lathe Spinner TC600-65 MC in the identified critical turning operation,

- Milling head WHN 13 CNC with milling head HUI 50 in the identified critical operation precision machining.

Subsequently, the quantification of time savings that can be achieved in clearly identified production operations and their critical points where the following savings of the working time fund can be achieved was performed:

- by implementing and using the LVD EasyForm9 220/30 press brake. it is possible to achieve savings of $31.14 \%$ of working time (Table 9 ), 
- implementation and use of CNC lathe Spinner TC600-65 MC can achieve savings of $24.27 \%$ (Table 10). while the quantification in question does not consider the compatibility of technical parameters in the production process, i.e. increasing time savings in product implementation,

- by implementing and using the WHN 13 CNC milling head with the HUI 50 MC milling head, it is possible to achieve a saving of $28.76 \%$ (Table 11), while the quantification in question does not take into account the compatibility of technical parameters in the production process. i.e. increase time savings in product implementation.

Table 9. Quantification of bending working time savings

\begin{tabular}{|c|c|c|c|c|c|c|c|c|}
\hline \multicolumn{9}{|c|}{ Save time when deploying LVD EasyForm9 220/30 } \\
\hline \multicolumn{9}{|c|}{ Average time per operation } \\
\hline Operation & 1 & 2 & 3 & 4 & 5 & 6 & 7 & 8 \\
\hline before & 3.41 & 10.3 & 10.27 & 1.73 & 2 & 1.17 & 0.67 & 14.81 \\
\hline after & 3.41 & 10.3 & 3.49 & 1.73 & 2 & 1.17 & 0.4 & 14.09 \\
\hline Operation & 9 & 10 & 4 & 6 & 7 & 8 & 9 & 10 \\
\hline before & 0.65 & 0.27 & 1.82 & 1.12 & 0.66 & 14.83 & 0.81 & 0.31 \\
\hline after & 0.22 & 0.27 & 1.82 & 1.12 & 0.4 & 14.83 & 0.28 & 0.31 \\
\hline Operation & 11 & 12 & & & & & SUM & \multirow{3}{*}{$\begin{array}{c}\text { Time saving } \\
\mathbf{3 1 . 1 4 \%}\end{array}$} \\
\hline before & 16.32 & 0.13 & & & & & 81.273 & \\
\hline after & 0 & 0.13 & & & & & 55.968 & \\
\hline
\end{tabular}

Source: Authors own elaboration.

Table 10. Quantification of bending working time savings

\begin{tabular}{|c|c|c|c|c|c|c|c|c|}
\hline \multicolumn{9}{|c|}{ Save time when using the Spinner TC 600-65 MC } \\
\hline \multicolumn{9}{|c|}{ Average time per operation } \\
\hline Operation & 1 & 2 & 3 & 4 & 5 & 6 & 7 & 8 \\
\hline before & 2.67 & 8.96 & 2.43 & 4.57 & 16.57 & 2.41 & 3.3 & 6.69 \\
\hline after & 2.67 & 8.96 & 2.43 & 4.36 & 15.79 & 1.39 & 2.31 & 0 \\
\hline Operation & 9 & 10 & 11 & 12 & 13 & 14 & SUM & \multirow{3}{*}{$\begin{array}{c}\text { Time saving } \\
24.27 \%\end{array}$} \\
\hline before & 2.07 & 3.36 & 2.03 & 3.21 & 0.37 & 0.47 & 60.957 & \\
\hline after & 0 & 2.35 & 0 & 3.21 & 0.37 & 0.47 & 46.164 & \\
\hline
\end{tabular}

Source: Authors own elaboration. 
Table 11. Quantification of precision machining time savings

\begin{tabular}{|c|c|c|c|c|c|c|c|c|}
\hline \multicolumn{9}{|c|}{ Time saving when using WHN 13 CNC with HUI 50 milling head } \\
\hline \multicolumn{9}{|c|}{ Average time per operation } \\
\hline Operation & 1 & 2 & 3 & 4 & 5 & 6 & 7 & 8 \\
\hline before & 31.03 & 50.836 & 11.88 & 61.53 & 10.93 & 7.972 & 17.493 & 9.026 \\
\hline after & 31.03 & 50.836 & 11.88 & 61.53 & 10.93 & 7.972 & 16.968 & 0 \\
\hline Operation & 9 & 10 & 6 & 7 & 8 & 9 & 10 & 6 \\
\hline before & 8.053 & 6.146 & 6.169 & 56.29 & 15.88 & 6.875 & 6.185 & 0.31 \\
\hline after & 0 & 0 & 6.169 & 54.6 & 0 & 0 & 6.185 & 0.31 \\
\hline Operation & 7 & 8 & 9 & 10 & 6 & 7 & 8 & 9 \\
\hline before & 35.27 & 13.373 & 8.115 & 13.35 & 7.728 & 36.1 & 12.195 & 8.3805 \\
\hline after & 34.21 & 0 & 0 & 0 & 7.728 & 35.02 & 0 & 0 \\
\hline Operation & 10 & 6 & 7 & 8 & 9 & 10 & 11 & 12 \\
\hline before & 10.88 & 6.365 & 32.25 & 15.18 & 8.092 & 14.38 & 11.34 & 3.121 \\
\hline after & 0 & 6.365 & 31.29 & 0 & 0 & 0 & 11.34 & 3.121 \\
\hline Operation & 13 & 14 & 15 & & & & SUM & \\
\hline before & 8.202 & 12.907 & 2.965 & & & & 577.27 & $\begin{array}{c}\text { Time saving } \\
28.76 \%\end{array}$ \\
\hline after & 8.202 & 12.907 & 2.965 & & & & 411.24 & \\
\hline
\end{tabular}

Source: Authors own elaboration.

From the above comparative quantifications of the proposed innovative measures. which were identified based on explicit quantifications of critical points of partial operations it can be stated that the use of the proposed innovative measures will determine the working time savings of $15.22 \%$ (Table 12).

Table 12. Quantification of the benefits of introducing innovative elements into the production process

\begin{tabular}{|c|c|c|}
\hline & The production process today & Proposed production process \\
\hline \multicolumn{3}{|l|}{ Beginning } \\
\hline Laser cutting & 336.332 & 336.332 \\
\hline Sawing & 41.478 & 41.478 \\
\hline Bending & $=2 \times 81.273=162.546$ & $=2 \times 55.968=111.936$ \\
\hline Turning & $=3 \times 60.957=182.871$ & $=3 \times 46.164=138.492$ \\
\hline Welding & 382.286 & 382.286 \\
\hline Precise machining & 577.268 & 411.242 \\
\hline Conservation & 31.859 & 31.859 \\
\hline \multicolumn{3}{|l|}{ End } \\
\hline \multirow[t]{2}{*}{ SUM in minutes } & 1714.64 & 1453.63 \\
\hline & Saved time: & $15.22 \%$ \\
\hline
\end{tabular}

Source: Authors own elaboration. 


\section{SUMMARY AND CONCLUSION}

Innovation of products, machine - technical equipment, or the whole technological process clearly contribute not only to maintaining the competitiveness of the company but also to its environmental responsibility in the exploitation of terrestrial and other natural resources in favour of the use of renewable resources and to reduce the emission of pollutants including $\mathrm{CO}$ and $\mathrm{CO}_{2}$ which also contribute to the implementation of the so-called low carbon technologies. Purchase of new machinery and equipment that can work in various production regimes not only increases the quality but also the energy demand. The merger must consider activities at individual workplaces into one continuous operation on machinery which will reduce the need for manipulation or operational adjustment of the machine and at the same time reaches min. 10\% savings energy in the flow of production. An important aspect is working in multiple axes or at different angles.

Such mechanical - technical equipment can during smooth operation control and self exchange reduce the time required for the final product by more than $15 \%$ over time production process. If processes increasing production efficiency while increasing labour fund savings time of human activity of the company will be based on innovation in production then the company will gain significant market position, reduce the negative impact on environmental quality and increase energy efficiency.

\section{REFERENCES}

Budzianowski, W. M. (2016). A review of potential innovations for production. conditioning and utilization of biogas with multiple-criteria assessment. "Renewable and Sustainable Energy Reviews", 54. DOI: 10.1016/j.rser.2015.10.054.

Carter, F. J., Jambulingam, T., Gupta, V. K., Melone, N. (2001). Technological innovations: a framework for communicating diffusion effects, "Information \& Management", 38(5). DOI:10.1016/s0378-7206(00)00065-3.

Damanpour, F., Gopalakrishan. (2001). The Dynamics of the Adoption of Product and Process Innovations in Organizations. "Journal of Management Studies", 38(1). DOI:10.1111/ 1467-6486.00227.

Dicová, J. (2008). Postavenie manažéra pri riadeni inovačných projektov [In:] Zborník z medzinárodnej vedeckej konferencie Marketing a obchod 2008, Zvolen 2008. Drevárska fakulta TU Zvolen.

Estélyiová, K. (2007). Podstata inovácií a ich využitie v malých a stredných podnikoch [online]. Brno:V ysoké učení technické v Brně [access: 18.03.2020]. Access on the internet: http://www.konference.fbm.vutbr.cz/workshop/papers/papers2007/estelyiova.pdf

Guan, J., Chen, K. (2010). Measuring the innovation production process: A cross-region empirical study of China's high-tech innovations. "Technovation", 30(5-6). DOI:10.1016/ j.technovation.2010.02.001.

Henriques, I., Sadorsky, P. (2007). Environmental technical and administrative innovations in the Canadian manufacturing industry. "Business Strategy and the Environment", 16(2). DOI: $10.1002 / \mathrm{bse} .475$.

Ivanová, E., Tomanová, M. (2014). Inovácie ako zdroj konkurencieschopnosti ekonomiky SR. Sociálno-ekonomická revue, ročník 12, číslo 01.

Kováč, M., Lešková, A., Sabadka, D., Kováčová, L. (2011). Tvorba a riadenie inovácií. Košice $T U-S j F$ (Original in Slovak). 
Malik, K., Bergfeld, M. (2015). A conceptual framework for intra-company technology transfer: cases of leveraging production process innovations across MNEs. "Technology Analysis \& Strategic Management”, 27(10). DOI:10.1080/09537325.2015.1060309.

Vivero, R. L. (2002). The impact of process innovations on firm's productivity growth: the case of Spain. „Applied Economics”, 34(8). DOI:10.1080/00036840010019684.

DOI: 10.7862/rz.2021.mmr.29

The text was submitted to the editorial office: December 2021.

The text was accepted for publication: December 2021. 
